\title{
Investigating Occipito-temporal Contributions to Reading with TMS
}

\author{
Keith J. Duncan ${ }^{1}$, Chotiga Pattamadilok ${ }^{1,2}$, and Joseph T. Devlin ${ }^{1}$
}

\begin{abstract}
The debate regarding the role of ventral occipito-temporal cortex (vOTC) in visual word recognition arises, in part, from difficulty delineating the functional contributions of vOTC as separate from other areas of the reading network. Here, we investigated the feasibility of using TMS to interfere with vOTC processing in order to explore its specific contributions to visual word recognition. Three visual lexical decision experiments were conducted using neuronavigated TMS. The first demonstrated that repetitive stimulation of vOTC successfully slowed word, but not nonword, responses. The second confirmed and extended these findings by demonstrating the effect was specific to vOTC and not present in the adjacent lateral occipital complex. The
\end{abstract}

\section{INTRODUCTION}

There is considerable debate regarding the role of left ventral occipito-temporal cortex (vOTC) during reading (Cai, Lavidor, Brysbaert, Paulignan, \& Nazir, 2008; Dehaene, Cohen, Sigman, \& Vinckier, 2005; Hillis et al., 2005; Cohen \& Dehaene, 2004; Price \& Devlin, 2003, 2004; McCandliss, Cohen, \& Dehaene, 2003). In part, this stems from a fundamental difficulty in identifying functional contributions from specific regions for tasks that engage highly distributed neural systems. For example, the claim that vOTC is the site of abstract orthographic representations is based on a combination of fMRI, ERP, and neuropsychological studies, all of which implicate vOTC in visual word recognition. Individually, however, no single line of evidence is particularly convincing. Consider the fMRI data showing that VOTC activation patterns for words are robust to changes in font, case, and retinal location (Cohen et al., $2000,2002)$. Although these are important properties of orthographic representations, the same patterns of activation are also seen in other left-hemisphere areas including the inferior frontal gyrus, inferior parietal lobule, and middle temporal cortex, making it difficult to attribute specific processing characteristic exclusively to vOTC (Price \& Devlin, 2004). Similarly, electrophysiological studies demonstrate that visual words activate vOTC approximately

${ }^{1}$ University College London, London, UK, ${ }^{2}$ Fonds de la Recherche Scientifique-FNRS and Université Libre de Bruxelles, Belgium final experiment used paired-pulse TMS to investigate the time course of vOTC processing for words and revealed activation starting as early as $80-120$ msec poststimulus onset-significantly earlier than that expected based on electrophysiological and magnetoencephalography studies. Taken together, these results clearly indicate that TMS can be successfully used to stimulate parts of vOTC previously believed to be inaccessible and provide a new tool for systematically investigating the information processing characteristics of vOTC. In addition, the findings provide strong evidence that lexical status and frequency significantly affect vOTC processing, findings difficult to reconcile with prelexical accounts of vOTC function.
170-200 msec poststimulus onset, suggesting that this activation may reflect the earliest stage of abstract orthographic processing (McCandliss et al., 2003; Cohen et al., 2000; Salmelin, Service, Kiesila, Uutela, \& Salonen, 1996; Nobre, Allison, \& McCarthy, 1994). Other ERP and MEG studies, however, reveal that vOTC activation is concurrent with activation in peri-sylvian regions (Pulvermüller et al., 2006; Pammer et al., 2004), again calling into question the unique processing characteristics of vOTC. Finally, lesions to vOTC can result in an acquired reading disorder called pure alexia (Gaillard et al., 2006; Leff et al., 2001; Dejerine, 1892), suggesting that this region may be the site of stored visual word forms (Gaillard et al., 2006; Cohen et al., 2002). Unfortunately for this argument, left inferior parietal lesions can also result in pure alexia (Philipose et al., 2007; Warrington \& Shallice, 1980). In short, it has been difficult to unambiguously delineate the specific information processing that VOTC contributes to reading because correlational measures such as PET, fMRI, ERP, and MEG do not distinguish contributions from coactivated areas and because of the well-known difficulties in interpreting neuropsychological studies. Consider, for example, the fact that the lateral occipital complex (LOC) is consistently activated during visual word recognition studies (Duncan, Pattamalidok, Kneirim, \& Devlin, 2009; Wright et al., 2008; Price et al., 2006) but damage to this region does not tend to result in reading deficits (Philipose et al., 2007). This discrepancy between the neuroimaging and neuropsychological literature highlights 
the need for a method to temporarily, and noninvasively, perturb the information processing in these regions to investigate the causal relations between activation and reading.

TMS would seem to offer such a tool (Sack, 2006; PascualLeone, Bartres-Faz, \& Keenan, 1999; Barker \& Jalinous, 1985), but it is widely believed that the location of vOTC on the ventral surface of the brain makes it inaccessible to TMS. Here, we explicitly tested this assumption in three TMS experiments using a lexical decision task to engage visual word recognition. The primary aim of the first experiment was to determine whether repetitive TMS (rTMS) to vOTC would interfere with visual word recognition. A second goal was to determine whether a similar effect would be seen for LOC stimulation. The second experiment further investigated potential differences between vOTC and LOC contributions to reading using rTMS, whereas the third experiment used paired-pulse TMS to delineate the time course of processing in left vOTC during reading.

\section{METHODS}

\section{Participants}

Sixty-four subjects (35 men, 29 women, aged 18 to 45 years, mean $=27$ years) participated in three experiments. All were right-handed, native English speakers with normal or corrected-to-normal vision. None had any form of dyslexia, a personal history of neurological disease, or a family history of epilepsy according to self-reports. Each gave informed consent after the experimental procedures were explained. The experiments were approved by the Berkshire NHS Research Ethics Committee.

\section{Functional Imaging}

All subjects participated in a single fMRI session whose purpose was to functionally localize two areas of occipitotemporal cortex for neuronavigated stimulation. These were a region of vOTC sensitive to reading visual words that is typically located in the posterior occipito-temporal sulcus (Duncan et al., 2009; Wright et al., 2008; BenShachar, Dougherty, Deutsch, \& Wandell, 2007) and a lateral region sensitive to visual objects, often called the LOC (Grill-Spector et al., 1999; Malach et al., 1995). The functional localizer scan used a 1-back task with four categories of visual stimuli: written words, pictures of common objects, scrambled pictures of the same objects, and consonant letter strings. Subjects were instructed to press a button if the stimulus was identical to the preceding stimulus and $12.5 \%$ of the stimuli were targets. A block design was used to maximize statistical sensitivity. Each block consisted of 16 trials from a single category presented one every second. A trial began with a 650-msec fixation cross followed by the stimulus for 350 msec. In between blocks, subjects viewed a fixation cross for 16 sec. Each run entailed six blocks per category and all subjects participated in two runs with the order counterbalanced across subjects. In total, there were 192 stimuli per category.

Whole-brain imaging was performed on a Siemens 1.5-Tesla MR scanner at the Birkbeck-UCL Centre for Neuroimaging (BUCNI) in London. The functional data were acquired with a gradient-echo EPI sequence $(\mathrm{TR}=$ $3 \mathrm{sec}, \mathrm{TE}=50 \mathrm{msec}, \mathrm{FOV}=192 \times 192$, matrix $=64 \times$ 64) giving a notional resolution of $3 \times 3 \times 3 \mathrm{~mm}$. In addition, a high-resolution anatomical scan was acquired (T1-weighted FLASH, TR $=12 \mathrm{msec}, \mathrm{TE}=5.6 \mathrm{msec}$, $1 \mathrm{~mm}^{3}$ resolution) for anatomically localizing activations in individuals.

Image processing was carried out using FSL 4.0 (www. fmrib.ox.ac.uk/fsl). To allow for T1 equilibrium, the initial two images of each run were discarded. The data were then realigned to remove small head movements (Jenkinson, Bannister, Brady, \& Smith, 2002), smoothed with a 6-mm FWHM Gaussian kernel, and prewhitened to remove temporal autocorrelation (Woolrich, Ripley, Brady, \& Smith, 2001). The resulting images were entered into a general linear model with four conditions of interest corresponding to the four categories of visual stimuli. Blocks were convolved with a double gamma "canonical" hemodynamic response function (Glover, 1999) to generate the main regressors. In addition, the estimated motion parameters were entered as covariates of no interest to reduce structured noise due to minor head motion. Linear contrasts of [words $>$ fixation] and [objects $>$ scrambled objects] identified reading- and object-sensitive areas, respectively. First-level results were registered to the MNI-152 template using a 12-DOF affine transformation (Jenkinson \& Smith, 2001), and a subsequent second-level, fixed-effects model combined the two first-level runs into a single, subject-specific analysis. This was then transformed into the participant's native structural space and used to target stimulation in the TMS experiment.

\section{Experiment 1}

The aims of the first experiment were to first determine whether TMS could be successfully used to stimulate vOTC and interfere with visual word recognition, and second to investigate the contributions of vOTC and LOC to reading. Fourteen participants ( 7 men, 7 women, aged 19 to 38 years, mean $=25$ years) performed a visual lexical decision task while rTMS was pseudorandomly delivered to one of three target sites.

The lexical decision experiment consisted of four conditions, each comprising 100 items: low-frequency (LF) words (1-10 occurrences per million), high-frequency (HF) words (20-650 occurrences per million), pronounceable pseudowords (e.g., "glats"), and unpronounceable consonant letter strings (e.g., "btfj"). Frequency values were obtained from the CELEX database of British-written English (Baayen, Piepenbrock, \& Van Rijn, 1993). LF and 
HF items were treated separately because imaging studies have shown greater vOTC activation for LF than HF words (Bruno, Zumberge, Manis, Lu, \& Goldman, 2008; Kronbichler et al., 2004; Kuo et al., 2003), suggesting that stimulation of this region may have a larger effect on LF words. In addition, two nonlexical conditions were included because imaging studies have also shown that pseudowords elicit either equivalent or greater vOTC activation than words (Mechelli, Gorno-Tempini, \& Price, 2003; Rumsey et al., 1997; Price, Wise, \& Frackowiak, 1996; Nobre et al., 1994), and thus, stimulation of this region may affect both legal words and pronounceable nonwords. Consequently, consonant letter strings were included as an additional control condition where stimulation was not expected to impair performance. To avoid repeating stimuli within a testing session, five versions of the lexical decision were created (each comprising 20 items per condition) and matched for written word frequency (overall and separately for both LF and HF items) (Baayen et al., 1993), rated familiarity (Coltheart, 1981), letter length, number of syllables, and bigram frequency. The order of the versions was balanced across subjects and stimulation sites. In addition, an independent set of items was used for practice.

rTMS was pseudorandomly delivered on half of all trials. Pulses were delivered at 100, 200, 300, 400, and $500 \mathrm{msec}$ poststimulus onset (i.e., $10 \mathrm{~Hz}$ for $500 \mathrm{msec}$ ). The intensity was set to $110 \%$ of the subject's motor threshold as measured by a visible twitch of the hand. Typically, this measure is approximately $10 \%$ higher than motor thresholds measured with motor-evoked potentials and, therefore, is a very conservative measure in the sense that it ensured sufficient intensity to stimulate motor cortex. This value was increased by an additional 10\% in the main experiment to ensure sufficient intensity to reach vOTC. Even so, this was well within established safety limits (Wassermann, 1998; Wassermann et al., 1996) and this general protocol has been widely used to temporarily interfere with processing in relatively focal cortical zones (Manenti, Cappa, Rossini, \& Miniussi, 2008; Pitcher, Garrido, Walsh, \& Duchaine, 2008; Sandrini, Rossini, \& Miniussi, 2008; Skarratt \& Lavidor, 2006; Gough, Nobre, \& Devlin, 2005; Göbel, Walsh, \& Rushworth, 2001; Rushworth, Ellison, \& Walsh, 2001). In summary, then, the experiment used a within-subject design with site (vOTC, vertex, LOC), stimulus (LF words, HF words, pseudowords, consonants), and TMS (rTMS, none) as independent factors.

\section{Procedure}

Each trial began with a fixation cross displayed for 500 msec, followed by a visual letter string for $200 \mathrm{msec}$ and then a blank screen for $2300 \mathrm{msec}$, giving a total duration of $3 \mathrm{sec}$. Subjects indicated whether the letter string formed a real word in English or not by pressing a button using either their right or left index finger. Responses were fully counterbalanced for response hand across subjects. Accuracy and reaction times (RTs) were recorded.

The three stimulation sites were tested sequentially in a single session with their order counterbalanced across subjects. A session began by measuring the participant's motor threshold using single pulses of TMS delivered to the hand area of left primary motor cortex. The participant then performed a practice session of the lexical decision experiment without any TMS to familiarize them with the task. Next, one of the three testing sites was chosen and the participant was introduced to the sensation of rTMS at that site. For both vOTC and LOC, the coil was held behind the left ear and, therefore, the sound of the coil discharge was prominent even though all participants used an earplug in their left ear to attenuate this noise. In addition, stimulation affected the temporalis muscle and produced a unilateral facial twitch. When asked afterward, participants were unable to distinguish the sensation of stimulation from the two temporal lobe sites. In contrast, stimulation of the vertex was farther from the ear, did not produce any muscle twitches, and was easily distinguished. After familiarization with the sensation, the participant performed a practice lexical decision run with rTMS to get used to performing the task with concurrent rTMS. Finally, they completed the lexical decision experiment for the given site using one of the five stimulus versions. The procedure was then repeated for the other two testing sites using different stimulus versions. At each location, including finding the motor threshold, the location and orientation of the coil were recorded for later analyses.

A Magstim Rapid ${ }^{2}$ stimulator (Magstim, Whitland, UK) with a 70-mm figure-of-eight coil was used to deliver the stimulation. In addition, a frameless stereotaxy system (Brainsight software; Rogue Research, Montreal, Canada) was used with a Polaris Vicra infrared camera (Northern Digital, Waterloo, Ontario, Canada) to accurately target stimulation and to measure the distance between the coil and the stimulation site. vOTC and LOC sites were defined based on the individual's fMRI data. The hand area of motor cortex was identified anatomically according to the method of Yousry et al. (1997) and was verified by finding the location which produced a twitch in the right hand. The distance from the scalp to these three targets was measured for each subject to evaluate differences relating to accessibility. In each case, the distance between the target and the scalp was measured using Brainsight along the trajectory of stimulation.

\section{Experiment 2}

The aim of the second experiment was to investigate the anatomical specificity of the interference effect and clarify an ambiguous finding from the previous experiment by determining whether stimulation of LOC interfered with visual word recognition or not. Twenty-six subjects (13 men, 13 women, aged 18 to 45 years, mean $=26$ years $)$ 
performed a visual lexical decision experiment similar to the first. The primary differences were that (i) this experiment used a between-subject design with 13 participants per stimulation site; (ii) only two sites were tested (vOTC and LOC); and (iii) only two stimulus conditions were included (LF words and pseudowords), each containing 40 items and the same stimuli were used for both sites. There were two versions whose order was counterbalanced across the stimulation sites. The stimuli were a subset of those used in the previous experiment and were matched across version for written word frequency, rated familiarity, letter length, number of syllables, and bigram frequency. Finally, the TMS intensity was set to 100\% of motor threshold rather than $110 \%$ as in the previous experiment. Otherwise, the procedures were the same as those above.

\section{Experiment 3}

The aim of the final experiment was to investigate the time course of vOTC involvement in reading using paired-pulse TMS. Twenty-four participants (15 men, 9 women, aged 19 to 46 years, mean $=29$ years) performed a visual lexical decision experiment in which every trial had two pulses of TMS separated by 40 msec. Subjectively, this feels like a single pulse but it induces a more robust interference effect (O’Shea, Johansen-Berg, Trief, Gobel, \& Rushworth, 2007; Pitcher, Walsh, Yovel, \& Duchaine, 2007; Juan \& Walsh, 2003). This experiment also used a betweensubject design, with one group of 12 participants receiving TMS over vOTC and the other over the vertex. Within each group, there were five different timing conditions with pulses at either 0 and $40 \mathrm{msec}, 40$ and $80 \mathrm{msec}, 80$ and $120 \mathrm{msec}, 120$ and $160 \mathrm{msec}$, or 160 and $200 \mathrm{msec}$ poststimulus onset, and the order was pseudorandomized. All trials had TMS in one of the five timing windows. All timing conditions included 20 LF words and 20 pseudowords and were matched for written word frequency, rated familiarity, letter length, number of syllables, and bigram frequency. The five versions of the stimuli contained the same 200 items but each set of 40 items was rotated across each of the five timing conditions, in addition to being matched for written word frequency, rated familiarity, letter length, number of syllables, and bigram frequency. TMS intensity was set to $100 \%$ of the subject's motor threshold and the blank screen between trials was reduced from 2300 to $1300 \mathrm{msec}$, giving a total trial duration of 2 sec. Otherwise, all procedures were the same as those in the previous experiments.

\section{Analyses}

RTs were measured from the onset of the target. Responses times shorter than $300 \mathrm{msec}$ or longer than $1500 \mathrm{msec}$ were trimmed, amounting to $0.2 \%, 0.0 \%$, and $0.2 \%$ of the data in Experiments 1 to 3, respectively. To minimize the effect of outliers in the RT data, median RTs for correct responses per condition per subject were used in the statistical analyses (Ulrich \& Miller, 1994). ANOVAs were used to test for effects of interest, and two-tailed paired $t$ tests were used with Bonferroni corrections for multiple comparisons unless stated otherwise.

In our experience, the $10-\mathrm{Hz}, 500-\mathrm{msec}$ stimulation protocol rarely shows disruption effects of more than $100 \mathrm{msec}$ and, moreover, the effect is typically specific to a particular subset of conditions (i.e., not the control stimuli, task, or site). Thus, when inhibitory TMS effects larger than 100 msec are observed and are present across conditions, these are generally attributed to nonspecific effects of TMS such as: (i) anxiety about the stimulation, (ii) a priori "knowledge" that TMS slows responses, or (iii) strong peripheral muscle stimulation that the participant finds impossible to ignore. In all three cases, the effect size and distribution across conditions is clearly different from the normal pattern and is easily identified and excluded. Data from three participants fit these criteria for physiologically implausible TMS effects. In the first experiment, one participant had RT increases for vOTC stimulation that were, on average, 173 msec slower than no-TMS trials across all conditions. Two participants in the second experiment showed TMS effects of +216 and +201 msec. In both cases, these were more than double the next largest effect, over 100 msec outside the range of the other participants, and present across conditions, suggesting they were primarily due to peripheral, rather than central, effects of TMS. Consequently, these data were excluded from further analyses.

\section{RESULTS \\ Experiment 1}

Because vOTC is generally regarded as inaccessible to TMS due to its depth, we began by explicitly measuring the distance from the scalp to the most highly activated voxel within vOTC and LOC used to target the stimulation. The distance was measured along the trajectory of the magnetic field and, therefore, reflects the distance between the coil and the stimulated region of cortex (Figure 1). In addition, the distance to the hand area of primary motor cortex was measured as a comparison. Because we did not functionally localize this using fMRI, the "omega knob" was marked independently by two of the authors (K. J. D. and J. T. D.) using anatomical criteria (Yousry et al., 1997) and the midpoint of these voxels was used to compute the distance from the scalp along the stimulation trajectory. The results for all subjects are shown in Table 1 . On average, the depth of vOTC was $26.3 \mathrm{~mm}$, which was not significantly different from the M1 hand area at $27.2 \mathrm{~mm}$ $[t(13)=1.0, p=.684]$, suggesting the two are equally accessible to TMS. LOC, on the other hand, was significantly closer to the scalp than M1 with an average depth of only $20.5 \mathrm{~mm}[t(13)=5.2, p=.000]$, confirming its accessibility. It is worth noting, however, that because 
Figure 1. Sites and trajectories of TMS stimulation. Because the trajectories do not necessarily correspond to canonical orientations, these slices were chosen from three different participants as they were closest to coronal views, and thus, most familiar. On each slice, the target voxel is marked with a filled circle along the trajectory (gray line) of maximum stimulation. A second gray line outside of the head indicates the orientation of the coil. The depth ( $\mathrm{mm}$ ) measured from the scalp to the stimulation target is marked with an arrow. In addition, a number

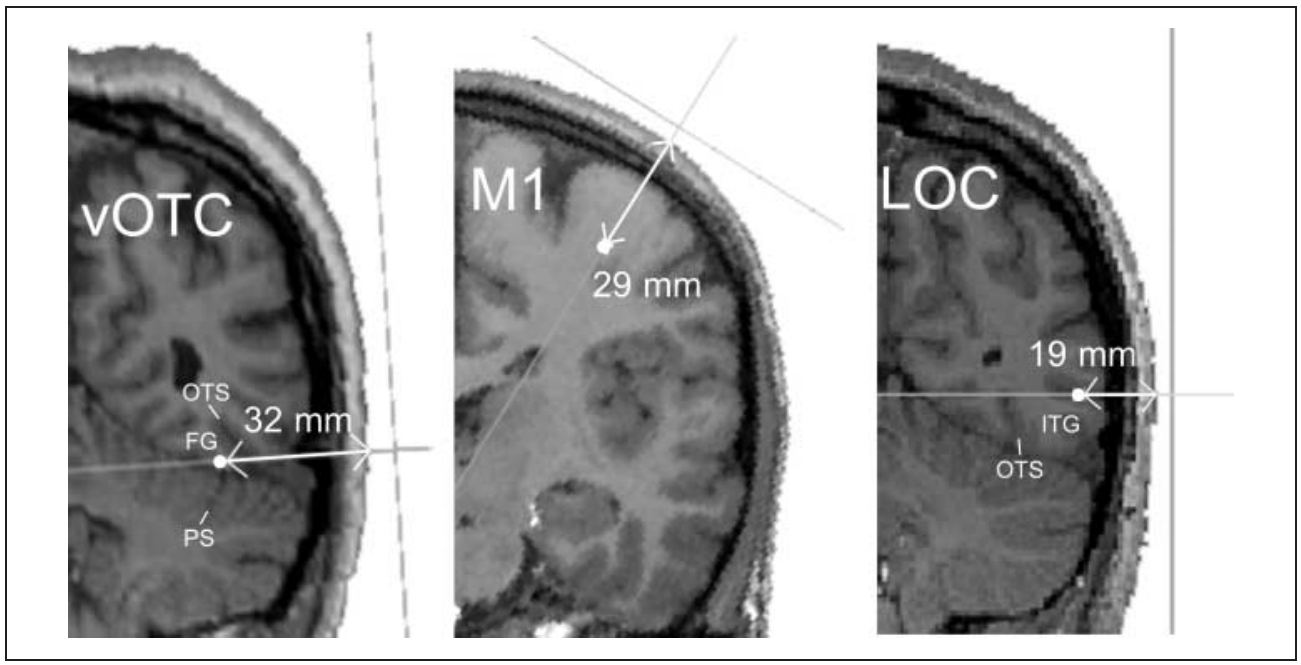
of anatomical landmarks are labeled: FG = fusiform gyrus; OTS = occipito-temporal sulcus; PS = principal sulcus of the cerebellum; and ITG $=$ inferior temporal gyrus. Note the trajectory of stimulation for vOTC involves minimal cerebellar and inferior temporal gyrus stimulation. Images are not to scale.

M1 was localized anatomically, whereas vOTC and LOC were localized with fMRI, the depth measurements are not fully compatible. Even so, the results suggest that the depth of vOTC is roughly equal to that of M1.

In the main lexical decision experiment, overall accuracy levels were 95\%, indicating that subjects had no difficulty performing the task. Accuracy scores were entered into a $3 \times 4 \times 2$ repeated measures ANOVA examining the effects of site (vOTC, vertex, LOC), condition (consonant strings, pseudowords, LF words, HF words), and TMS (rTMS, none). The only significant main effect was for condition $[F(3,39)=14.0, p=.000]$, indicating that subjects were significantly less accurate for pseudowords than HF words $[t(13)=6.0, p=.01]$ and consonant strings $[t(13)=7.4, p=.003]$ but only numerically less for LF words $[t(13)=2.6, p=.478]$. There was no main effect of TMS and no significant interactions, indicating that the presence of TMS did not affect accuracy.

The RT results are shown in Figure 2 and were analyzed with the same omnibus ANOVA. There was a main effect of condition $[F(3,39)=51.1, p=.000]$ indicating that, as expected, the four types of stimuli differed in difficulty, with consonant letters strings the easiest (471 msec), followed by HF words ( $517 \mathrm{msec}$ ), LF words (550 msec), and then pseudowords (624 $\mathrm{msec})$. In addition, there was a significant Condition $\times$ TMS interaction $[F(3,39)=$ $5.5, p=.003$ ] demonstrating that, on average, rTMS de- creased RTs across stimulation sites for pseudowords (634 vs. $613 \mathrm{msec}$ ) but increased RTs for LF words (543 vs. $558 \mathrm{msec}$ ). Interestingly, the facilitation effect on pseudowords was consistent for all three sites, whereas TMS interfered with LF words at the vOTC and LOC sites but facilitated responses at the vertex. Surprisingly, the threeway interaction was not significant, probably due to insufficient power given the complexity of the design (i.e., four stimulus conditions and three testing sites). Even so, Figure 2 shows that the response profiles to TMS were different across sites and, consequently, we chose to investigate these further.

In order to investigate these difference response profiles, we conducted a two-way repeated measures ANOVA for each site. The main effect of condition was present for all sites. For the vOTC site, there was no main effect of TMS $[F(1,13)=0.6, p=.449]$ but a significant Condition $\times$ TMS interaction $[F(3,39)=3.7, p=.021]$. Planned comparisons revealed that TMS selectively slowed responses to LF words $[+34$ msec, $t(13)=2.8, p=.017]$ without significantly affecting the other conditions (Figure 2A). The response profile for stimulation of the vertex, on the other hand, looked very different. Here, there was a significant main effect of TMS $[F(1,13)=6.2, p=.027]$ but no significant interaction $[F(3,39)=1.8, p=.160]$. Planned comparisons showed a significant TMS-induced speed-up for both pseudowords [ $-34 \mathrm{msec}, t(13)=2.7, p=.018]$

Table 1. Distances $(\mathrm{mm})$ from Scalp to Target Stimulation Site

\begin{tabular}{|c|c|c|c|c|c|c|c|c|c|c|c|c|c|c|c|}
\hline \multirow{2}{*}{$\frac{\text { Region }}{\text { vOTC }}$} & \multicolumn{14}{|c|}{ Participants } & \multirow{2}{*}{$\frac{\text { Mean (SEM) }}{26(0.9)}$} \\
\hline & 24 & 32 & 29 & 26 & 28 & 25 & 31 & 22 & 30 & 26 & 25 & 21 & 21 & 28 & \\
\hline M1 & 25 & 33 & 29 & 25 & 28 & 26 & 31 & 21 & 30 & 25 & 25 & 29 & 30 & 24 & $27(0.9)$ \\
\hline LOC & 23 & 26 & 17 & 20 & 21 & 21 & 28 & 23 & 17 & 13 & 21 & 16 & 19 & 23 & $21(1.1)$ \\
\hline
\end{tabular}




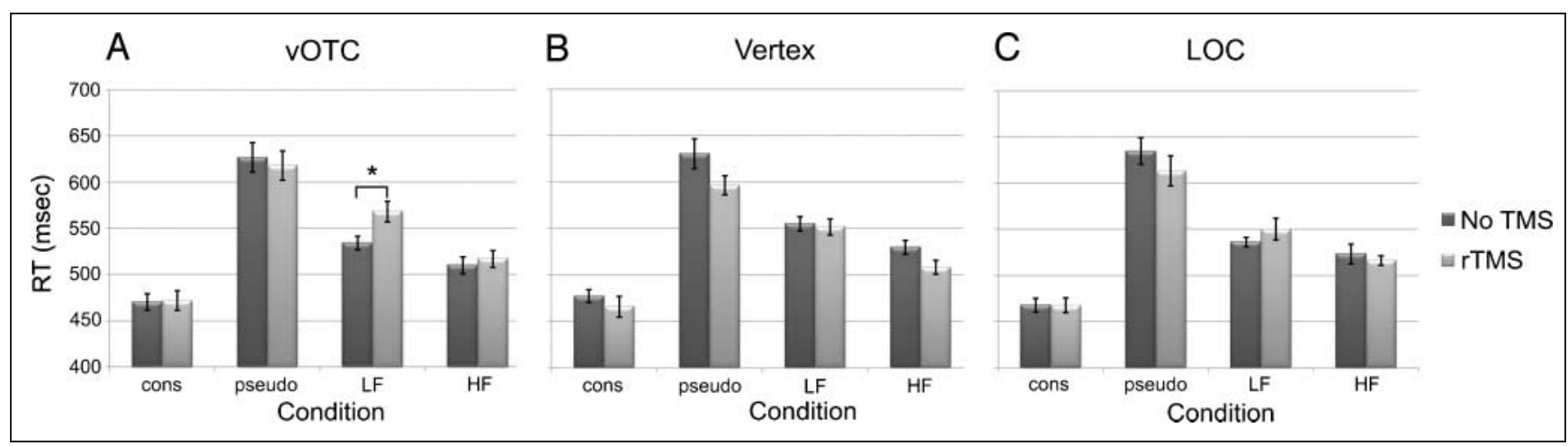

Figure 2. Mean RTs (msec) for rTMS to (A) vOTC, (B) vertex, and (C) LOC across the four stimulus conditions in Experiment 1 for no TMS (dark bars) and TMS (light bars). Cons = consonant letter strings; Pseudo = pronounceable pseudowords; LF $=$ low-frequency words; HF $=$ high-frequency words. Error bars represent standard error of the mean, adjusted to accurately reflect the variance in a repeated measures design (Loftus \& Masson, 1994). * indicates a significant TMS effect at $p<.05$.

and HF words [ -24 msec, $t(13)=2.9, p=.012]$, although both LF words and consonants were numerically faster as well (Figure 2B). In other words, TMS of the vertex appeared to have a nonspecific, intersensory facilitation effect (Terao et al., 1997). Finally, stimulation at LOC produced neither a significant main effect of TMS $[F(1,13)=$ $0.6, p=.458]$ nor an interaction $[F(3,39)=1.5, p=.217]$, although LF words showed a nonsignificant slowdown of +14 msec $[t(13)=1.0, p=.327]$ similar to that seen in vOTC (Figure 2C).

In summary, Experiment 1 demonstrated that stimulation of vOTC selectively slowed response times to LF words. This pattern was noticeably different than the nonspecific facilitation effect seen for vertex stimulation but qualitatively similar to the pattern at LOC. Given that both vOTC and LOC are activated by visual words (Duncan et al., 2009; Price et al., 2006; Moore \& Price, 1999) and that the two stimulation sites were only $2 \mathrm{~cm}$ apart, it is unclear whether there was an actual difference in the effects of TMS between these sites or whether the experiment was underpowered to detect a TMS effect at LOC. Consequently, the second experiment was designed to specifically address this question. To increase statistical sensitivity, the number of stimuli per condition was doubled and lexical items only consisted of LF words as these produced the largest TMS effect in Experiment 1. In addition, we chose to reduce the stimulation intensity from 110\% to
$100 \%$ of motor threshold based on the similar depths of M1 and vOTC. This helped to reduce the discomfort that some participants experienced during stimulation without reducing the likelihood of successfully stimulating the region.

\section{Experiment 2}

Like the first experiment, overall accuracy was high (94\%). Accuracy scores were entered into a three-way mixed ANOVA where the within-subjects factors were condition (words, pseudowords) and TMS (rTMS, no) and the between-subjects factor was site (vOTC, LOC). The only significant main effect was for TMS $[F(1,22)=7.7, p=$ $.011]$, reflecting a small TMS-induced decrease in accuracy (from 95\% to 93\%) that was present across sites and conditions. This is likely due to the peripheral effects of TMS stimulation, which was identical across the two occipitotemporal sites.

The critical results, however, concerned the TMS effect on RTs. To investigate this, the RT results (shown in Figure 3) were analyzed with the same omnibus ANOVA. There was a main effect of condition $[F(1,22)=43.3, p=.000]$, once again indicating that pseudowords (646 msec) were more difficult than LF words (585 msec). More importantly, the three-way interaction $[F(1,22)=8.0, p=.010]$ was clearly significant. Further analyses showed that vOTC stimulation
Figure 3. Mean RTs (msec) for (A) LF words and (B) pseudowords in Experiment 2. The only significant TMS effect was a +40 msec slow down for words with vOTC stimulation (for details, see Figure 2).

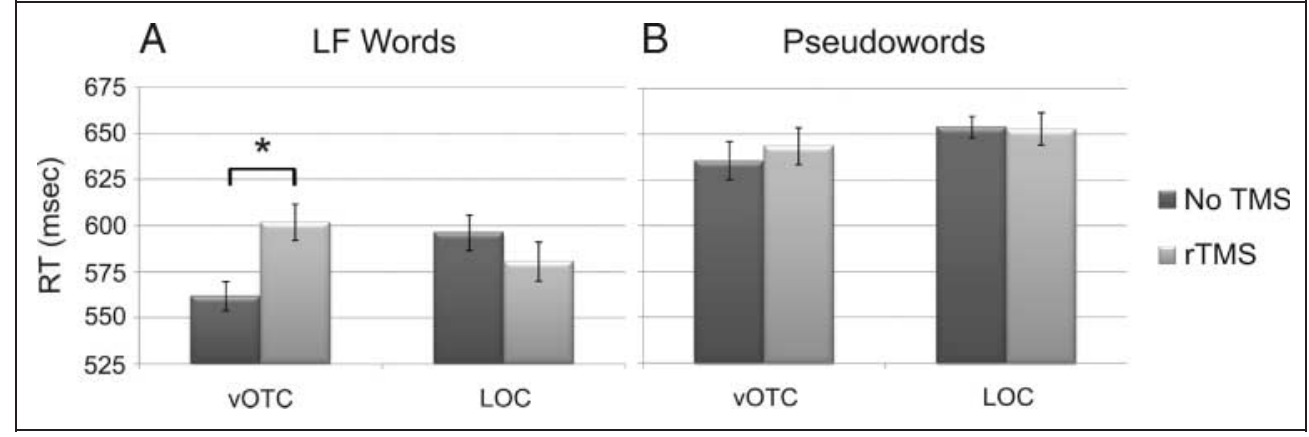


significantly slowed RTs for words $[+40 \mathrm{msec}, t(12)=3.1$, $p=.010]$ but had no effect on pseudowords [+8 $\mathrm{msec}$, $t(12)=0.7, p=.521]$. In contrast, LOC stimulation did not significantly affect RTs for words $[-15 \mathrm{msec}, t(12)=$ $1.0, p=.343$ ] or pseudowords [ $-1 \mathrm{msec}, t(10)=0.070$, $p=.945]$.

These results confirm that stimulation of vOTC interferes with reading $\mathrm{LF}$ words and demonstrates that the effect is present even at a lower stimulation intensity. Critically, the findings help to clarify the ambiguous results in the first experiment regarding the effects of LOC stimulation. Despite the greater sensitivity in Experiment 2, rTMS over LOC had no significant effect on LF words - in fact, if anything, LOC stimulation slightly reduced RTs for wordsconfirming that the interference seen for vOTC stimulation is specific to that site and not a general feature of occipitaltemporal stimulation. In other words, these peripheral stimulation effects cannot explain the current pattern of results because vOTC stimulation did not affect all stimuli equally; specifically, it inhibited word responses without affecting pseudowords. In addition, these effects were not present in LOC where stimulation produced essentially identical peripheral effects. In summary, then, these results demonstrate that vOTC stimulation successfully and selectively disrupted visual word recognition, whereas LOC stimulation did not. Consequently, the final experiment did not include LOC as a testing site because stimulation did not affect reading performance but, nonetheless, involved some discomfort due to peripheral enervation of the temporalis muscle.

\section{Experiment 3}

Like the previous experiments, accuracy was high with an average score of $94 \%$ across conditions. Although pseudowords were included in the task to ensure that participants correctly performed the lexical decision task, they were not included in the analyses as the previous experiments showed no effect of TMS on pseudowords. The word accuracy data were entered into a two-way mixed ANOVA,

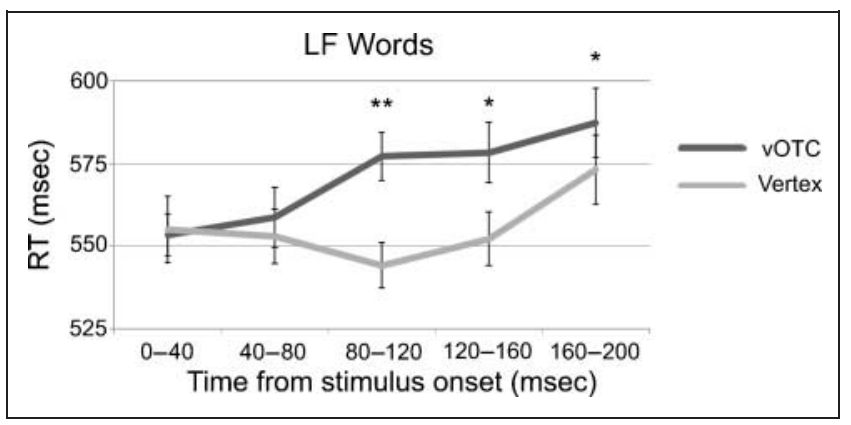

Figure 4. Mean RTs (msec) for TMS of vOTC (dark bars) and vertex (light bars) over five time windows, with error bars representing corrected standard error of the means (Loftus \& Masson, 1994). ** indicates a significant difference for vOTC stimulation from the 0 to 40 msec time window at $p<.01 ; *$ indicates $p<.05$. where the within-subjects factor was time $(0 / 40,40 / 80$, 80/120, 120/160, 160/200 msec poststimulus onset) and the between-subjects factor was site (vOTC, vertex). There were no significant main effects of time $[F(4,88)=1.6$, $p=.184]$ or site $[F(1,22)=0.3, p=.564]$ and no interaction $[F(4,88)=1.6, p=.174]$, indicating that TMS, once again, did not affect accuracy.

RTs for words are shown in Figure 4. Here, the 0$40 \mathrm{msec}$ time window served as the baseline condition (per testing site), as TMS was not expected to influence reading that early. A two-way mixed ANOVA revealed a main effect of time $[F(4,88)=4.2, p=.004]$ but no main effect of site $[F(1,22)=0.44, p=.514]$ or Site $\times$ Time interaction $[F(4,88)=1.9, p=.110]$. Visual inspection of Figure 4 suggests that the absence of interaction may be caused by an unexpectedly long RT for vertex stimulation in the 160-200 msec time window ( $573 \mathrm{msec}$ ) compared to the other time windows (range $=544-555 \mathrm{msec}$ ). The +18 msec effect, however, did not represent a significant slowdown relative to the $0-40$ window $[t(11)=1.6, p=$ $.145]$, consistent with the results of Experiment 1 and with the functional neuroimaging literature, neither of which demonstrates vertex involvement in reading at any level. As a result, we chose to exclude the 160-200 msec time window from further analyses.

When this time window was excluded, there was no main effect of time $[F(3,66)=1.1, p=.369]$ or site $[F(1,22)=0.5, p=.501]$ but a significant Site $\times$ Time interaction $[F(3,66)=2.9, p=.044]$. Planned comparisons used Bonferroni-corrected, one-tailed $t$ tests because the previous experiments showed that TMS to vOTC slowed, rather than speeded, responses. These indicated no significant effect at 40-80 msec $[t(11)=0.6, p=.8205]$ but significantly slowed responses for the 80-120 [+24 msec, $t(11)=3.4, p=.009]$ and $120-160 \mathrm{msec}[+25 \mathrm{msec}$, $t(11)=2.6, p=.0375]$ time windows. In contrast, there were no significant effects for stimulation of the vertex at any of the time windows [all $t(11)<1.3, p>.72$ ]. Figure 4 , therefore, illustrates three points. First, the earliest time window where TMS interfered with reading was 80120 msec poststimulus onset; second, this effect was also present in the subsequent time window of 120-160 msec; and third, that this interference effect was specific to the vOTC site.

\section{DISCUSSION}

The initial question we set out to investigate was whether it was possible to stimulate a left ventral occipital-temporal region involved in visual word recognition. The results of three independent experiments convincingly demonstrate that TMS can be used to successfully stimulate vOTC and interfere with reading. Although the subjective experience of TMS of vOTC and vertex is noticeably different, it is unlikely that the disruption in word reading was a general, peripheral effect of TMS given that the impairment was 
condition-specific and replicated. Moreover, this possibility is rendered even less likely if we consider the difference between vOTC and LOC stimulation (Experiment 2), given that the sensation of TMS is not noticeably different between these two posterior temporal lobe sites. Specifically, stimulation of LOC led to a (nonsignificant) increase in RTs for LF words that became a (nonsignificant) decrease in the second. The lack of any significant modulation by TMS and the fact that RT changes were not even in a consistent direction suggests that TMS to LOC had no effect on reading LF words, which contrasts with the results of vOTC stimulation. These findings are consistent with the neuropsychological literature which suggests that left hemisphere lesions to either vOTC or the inferior parietal lobule-but not LOC—can result in preferential impairments for reading (Philipose et al., 2007; Binder \& Mohr, 1992; Damasio \& Damasio, 1983; Geschwind, 1965; Dejerine, 1892) and demonstrate that vOTC, but not LOC, is necessary for visual word recognition. These findings have both methodological and theoretical implications.

\section{Implications for TMS}

The specific region of vOTC associated with reading is typically centered on the occipito-temporal sulcus and spreads medially onto the posterior fusiform gyrus (Duncan et al., 2009; Wright et al., 2008; Ben-Shachar et al., 2007). This area lies at a complex junction between the inferior temporal and fusiform gyri, just superior and lateral to the principal fissure of the cerebellum (see Figure 1). As a result, it is possible to orient the TMS coil such that the line of maximum stimulation runs between the cerebellum and the inferior temporal gyrus, and targets the occipitotemporal sulcus. Due to the smoothly varying topology of the magnetic field generated by a figure-of-eight coil, there is likely to be some stimulation of both inferior temporal gyrus and of cerebellar lobule VI, but the maximum effect targets the occipito-temporal sulcus and the lateral posterior fusiform gyrus.

Perhaps surprisingly, this region is, on average, no deeper than the hand area of primary motor cortex, which is easily accessible with TMS (Rothwell, 1997; PascualLeone, Vallssole, Wassermann, \& Hallett, 1994; Barker \& Jalinous, 1985). Consequently, the stimulation intensity necessary to affect vOTC can be estimated based on motor thresholds. Here, we used a fairly conservative measure of motor threshold (i.e., a visible muscle twitch), but lower values based on motor-evoked potentials may be sufficient and would help to reduce both the auditory and somatosensory effects of stimulating this region. Although we used fMRI to functional localize the precise area of vOTC to stimulate in each participant, this was not strictly necessary. Pilot work indicated that it was possible to functionally localize the optimal stimulation site using short TMS experiments before proceeding to the main experiment as previously done for other stimulation sites (e.g., Taylor, Nobre, \& Rushworth, 2007; Gough et al., 2005; Walsh,
Ellison, Battelli, \& Cowey, 1998). In practice, however, some participants found the extra stimulation uncomfortable; consequently, we chose to localize the site using fMRI instead. Both methods were effective in localizing the target site and presumably significantly reducing the intersubject variability relative to targeting based on either standard space coordinates or the 10-20 electrode system (Sack et al., 2009; Sparing, Buelte, Meister, Paus, \& Fink, 2008).

A final point worth mentioning here concerns the spatial resolution of TMS given the clear dissociation between stimulation of vOTC and LOC, two occipito-temporal regions separated by approximately $2 \mathrm{~cm}$. Although it is well established that TMS effects from single pulses can spread via anatomical connections beyond the site of stimulation (Ilmoniemi et al., 1997; Paus et al., 1997), TMS appears to have an effective spatial resolution of approximately $1 \mathrm{~cm}$. That is, single pulses of stimulation delivered roughly $1 \mathrm{~cm}$ apart on the scalp evoke very different responses in both motor (Brasil-Neto, McShane, Fuhr, Hallett, \& Cohen, 1992) and premotor cortices (Schluter, Rushworth, Mills, \& Passingham, 1999). Even short trains of repetitive stimulation have been shown to produce double dissociations between regions separated by as little as $2 \mathrm{~cm}$ (Pitcher, Charles, Devlin, Walsh, \& Duchaine, 2009; Gough et al., 2005). Thus, the current findings of a clear dissociation between stimulation of vOTC and LOC, despite their anatomical proximity, are consistent with the growing literature suggesting that the functional resolution of the technique is sufficient to distinguish contributions from relatively focal patches of neocortex.

\section{Theories of vOTC Functioning}

It is informative to compare the current TMS findings with those from related neuroimaging studies, where there are both similarities and important differences. For instance, many imaging studies report little, if any, activation in vOTC for consonant letter strings, consistent with the finding that vOTC stimulation had essentially no effect on consonant letter strings. Similarly, several imaging studies have demonstrated that activation in vOTC is modulated by word frequency, with greater activation for LF than HF words (Bruno et al., 2008; Kronbichler et al., 2004; Kuo et al., 2003). Here, we observed a (nonsignificant) slow down for HF words of $+7 \mathrm{msec}$ and a larger (significant) effect on LF words $(+34 \mathrm{msec})$, consistent with the imaging findings. It is possible that with greater statistical sensitivity, we would be able to document a reliable, graded effect of TMS on different classes of orthographic stimuli, but the current findings certainly are consistent with the imaging literature. Pseudowords, on the other hand, typically evoke equal or greater vOTC activation than words (Mechelli et al., 2003; Rumsey et al., 1997; Price et al., 1996; Nobre et al., 1994), and yet, TMS did not significantly, or even consistently, affect responses to pseudowords. It is worth noting, however, that in a lexical 
decision task, participants make a different response for words ("yes") and pseudowords ("no"), which may contribute to the different TMS effects for these two types of stimuli. On the other hand, the same response confound is present in the imaging studies using lexical decision, so this alone cannot explain the apparent disconnect between the TMS and imaging results. Further studies will be necessary to determine whether TMS differentially affects words and pseudowords even when the response is matched across conditions such as in a naming task. The current findings that vOTC makes different contributions to words and nonwords, however, are consistent with previous imaging studies that also found reliable lexicality effects in vOTC (Devlin, Jamison, Gonnerman, \& Matthews, 2006; Fiebach, Gruber, \& Supp, 2005) and have potentially important implications for understanding vOTC contributions to reading.

How do these findings speak to theories of vOTC function? Lexical frequency (i.e., LF $>$ HF) and lexicality (i.e., words $>$ pseudowords) effects are both difficult to explain in terms of stored, prelexical representations (Dehaene et al., 2005; McCandliss et al., 2003; Cohen et al., 2000, 2002), particularly when the stimuli were matched on orthographic factors including bigram frequency. According to this account, neurons in vOTC have receptive fields for bigrams - that is, they are specialized for detecting particular visual stimulus characteristics - and are therefore insensitive to the specific response properties of the task. As a result, any stimuli composed of legal bigrams should be equally affected by TMS, with no difference between LF and HF words or pseudowords, irrespective of the response. Clearly, the current TMS results, as well as previous imaging findings (Bruno et al., 2008; Devlin et al., 2006; Fiebach et al., 2005; Kronbichler et al., 2004; Kuo et al., 2003), are inconsistent with this proposal. An alternate possibility is that vOTC is the site of stored lexical representations of visual words (Kronbichler et al., 2004). Although sufficient to explain the current TMS results, this account is incompatible with previous imaging evidence showing neural repetition priming effects for visually similar, but lexically distinct, word pairs such as "corner-corn" (Devlin et al., 2006). A different possibility is that vOTC acts as an interface, integrating bottom-up, visual form information (that is not specific to written words) with top-down, nonvisual properties of the stimulus such as its sound or meaning (Devlin et al., 2006; Hillis et al., 2005; Price \& Friston, 2005). Because LF items place greater processing demands than HF items, there is greater activation in vOTC and stimulation has a larger disruption effect. By this account, however, pseudowords could be considered extremely LF items. In this case, the additional processing demands reflect the search, and subsequent failure, to link the visual stimulus with its nonvisual properties (e.g., what is the meaning of "bocket"?) and lead to the greater vOTC activation seen for pseudowords (Mechelli et al., 2003; Rumsey et al., 1997; Price et al., 1996; Nobre et al., 1994). Stimulation of the region does not have a significant effect on behavior, however, because the integration process fails regardless of the TMS-induced disruption. If true, then this hypothesis predicts that TMS will not affect stimuli when this integration process fails, regardless of the response demanded by the task. Clearly, further work is needed to better evaluate all three of these theories.

Finally, what do the paired-pulse results reveal about the temporal nature of orthographic processing within vOTC? Numerous ERP and MEG studies demonstrate that both written words and pseudowords evoke an early midline occipital positivity at approximately $80-100 \mathrm{msec}$ poststimulus, followed by a left occipito-temporal negativity between 170 and 200 msec (Bentin, Mouchetant-Rostaing, Giard, Echallier, \& Pernier, 1999; Tarkiainen, Helenius, Hansen, Cornelissen, \& Salmelin, 1999; McCandliss, Posner, \& Givon, 1997; Salmelin et al., 1996). The initial component reflects activity in V1 and is common to all visual stimuli, whereas the second component is thought to arise in vOTC and distinguishes between orthographic and nonorthographic stimuli (Cohen et al., 2000; Bentin et al., 1999; Nobre et al., 1994). Thus, it is typically assumed that vOTC is activated by an excitatory feedforward volley of activity spreading ventrolaterally from V1 to V2 and V4 and then into vOTC at approximately 170-200 msec poststimulus onset (Dehaene et al., 2005; McCandliss et al., 2003). Our results, however, suggest that information is present in vOTC substantially earlier-possibly as early as 80 msec after the stimulus appears on the retina. Even if the first pulse anticipated the feedforward volley of action potentials, its disruptive effect is believed to last only 3040 msec (Corthout, Uttl, Walsh, Hallett, \& Cowey, 1999; Ilmoniemi et al., 1997; Amassian et al., 1989), which suggests a time lag between the TMS and ERP/MEG latency values. Interestingly, the TMS results better match those from multiunit recordings in awake monkeys. For instance, the onset latencies for action potentials in V1 are between 20 and 30 msec (Schroeder, Mehta, \& Givre, 1998; Givre, Schroeder, \& Arezzo, 1994; Maunsell \& Gibson, 1992), which match TMS-induced disruption (Corthout et al., 1999) but considerably anticipate the P80-100 component. Similarly, the initial action potentials from the ascending visual pathway appear in posterior inferotemporal cortex between 60 and 120 msec poststimulus onset (Chen et al., 2007; Schroeder et al., 1998; Richmond, Optican, Podell, \& Spitzer, 1987; Ashford \& Fuster, 1985), consistent with the current TMS findings but much earlier than the N170 component. In other words, the time at which TMS exerts its disruptive effect precedes the peak times reported in ERP/MEG experiments (Walsh \& Cowey, 2000). Presumably, this reflects the fact that these components arise from large-scale neuronal synchrony across activity in multiple structures and, therefore, lag behind the earliest wave of activity in any given structure (Walsh \& Cowey, 2000; Schroeder et al., 1998). As a consequence, chronometric TMS studies may offer a more accurate measure of absolute regional timings than ERP or MEG, despite their greater temporal resolution. 
In conclusion, we have demonstrated that TMS can be used to successfully stimulate parts of vOTC and interfere with visual word recognition. These experiments open the door for a systematic exploration of vOTC contributions to reading and its relation to other higher-order visual functions (Starrfelt \& Gerlach, 2007; Joseph, Cerullo, Farley, Steinmetz, \& Mier, 2006; Price \& Devlin, 2003). Similarly, although we have focused entirely on left vOTC, this same approach should be useful for investigating right vOTC, including the so-called fusiform face area and its contributions to face recognition (Kanwisher, McDermott, \& Chun, 1997; Haxby et al., 1994; Sergent, Ohta, \& Macdonald, 1992) and other categories of visual objects (Haxby et al., 2001; Haxby, Hoffman, \& Gobbini, 2000).

\section{Acknowledgments}

We thank Phillip Kelley and Iris Knierim for their help with data collection and also Marty Sereno for helpful discussions. This research was supported by the BBSRC (K. J. D.), "Rayonnement International," Université Libre de Bruxelles (C. P.), and the Wellcome Trust (J. T. D.).

Reprint requests should be sent to Keith J. Duncan, Cognitive, Perceptual and Brain Sciences, UCL, Gower Street, London, WC1E 6BT, UK, or via e-mail: k.duncan@ucl.ac.uk.

\section{REFERENCES}

Amassian, V. E., Cracco, R. Q., Maccabee, P. J., Cracco, J. B., Rudell, A., \& Eberle, L. (1989). Suppression of visual perception by magnetic coil stimulation of human occipital cortex. Electroencephalography and Clinical Neurophysiology_Evoked Potentials Section, 74, 458-462.

Ashford, J. W., \& Fuster, J. M. (1985). Occipital and inferotemporal responses to visual signals in the monkey. Experimental Neurology, 90, 444-466.

Baayen, R. H., Piepenbrock, R., \& Van Rijn, H. (1993). The CELEX Lexical Database [CD-ROM].

Barker, A. T., \& Jalinous, R. (1985). Non-invasive magnetic stimulation of human motor cortex. Lancet, 1, 1106-1107.

Ben-Shachar, M., Dougherty, R. F., Deutsch, G. K., \& Wandell, B. A. (2007). Differential sensitivity to words and shapes in ventral occipito-temporal cortex. Cerebral Cortex, 17, 1604-1611.

Bentin, S., Mouchetant-Rostaing, Y., Giard, M. H., Echallier, J. F., \& Pernier, J. (1999). ERP manifestations of processing printed words at different psycholinguistic levels: Time course and scalp distribution. Journal of Cognitive Neuroscience, 11, 235-260.

Binder, J. R., \& Mohr, J. P. (1992). The topography of callosal reading pathways. A case-control analysis. Brain, 115, 1807-1826.

Brasil-Neto, J. P., McShane, L. M., Fuhr, P., Hallett, M., \& Cohen, L. G. (1992). Topographic mapping of the human motor cortex with magnetic stimulation: Factors affecting accuracy and reproducibility. Electroencephalography and Clinical Neurophysiology-Electromyography and Motor Control, 85, 9-16.

Bruno, J. L., Zumberge, A., Manis, F. R., Lu, Z.-L., \& Goldman, J. G. (2008). Sensitivity to orthographic familiarity in the occipito-temporal region. Neuroimage, 39, 1988-2001.
Cai, Q., Lavidor, M., Brysbaert, M., Paulignan, Y., \& Nazir, T. A. (2008). Cerebral lateralization of frontal lobe language processes and lateralization of the posterior visual word processing system. Journal of Cognitive Neuroscience, 20, 672-681.

Chen, C.-M., Lakatos, P., Shah, A. S., Mehta, A. D., Givre, S. J., Javitt, D. C., et al. (2007). Functional anatomy and interaction of fast and slow visual pathways in macaque monkeys. Cerebral Cortex, 17, 1561-1569.

Cohen, L., \& Dehaene, S. (2004). Specialization within the ventral stream: The case for the visual word form area. Neuroimage, 22, 466-476.

Cohen, L., Dehaene, S., Naccache, L., Lehéricy, S., Dehaene-Lambertz, G., Hénaff, M. A., et al. (2000). The visual word form area. Spatial and temporal characterization of an initial stage of reading in normal subjects and posterior split-brain patients. Brain, 123, 291-307.

Cohen, L., Lehéricy, S., Chochon, F., Lemer, C., Rivaud, S., \& Dehaene, S. (2002). Language-specific tuning of visual cortex? Functional properties of the visual word form area. Brain, 125, 1054-1069.

Coltheart, M. (1981). The MRC psycholinguistic database. Quarterly Journal of Experimental Psychology, 33A, 497-505.

Corthout, E., Uttl, B., Walsh, V., Hallett, M., \& Cowey, A. (1999). Timing of activity in early visual cortex as revealed by transcranial magnetic stimulation. NeuroReport, 10, 2631-2634.

Damasio, A. R., \& Damasio, H. (1983). The anatomic basis of pure alexia. Neurology, 33, 1573-1583.

Dehaene, S., Cohen, L., Sigman, M., \& Vinckier, F. (2005). The neural code for written words: A proposal. Trends in Cognitive Sciences, 9, 335-341.

Dejerine, J. (1892). Contribution à l'étude anatomo-pathologique et clinique des différentes variétés cécité verbale. Comptes Rendus Hebdomaladaires des Séances et Mémoires de la Société de Biologie, 4, 61-90.

Devlin, J. T., Jamison, H. L., Gonnerman, L. M., \& Matthews, P. M. (2006). The role of the posterior fusiform gyrus in reading. Journal of Cognitive Neuroscience, 18, 911-922.

Duncan, K. J., Pattamalidok, C., Kneirim, I., \& Devlin, J. T. (2009). Consistency and variability in functional localisers. Neuroimage, 46, 1018-1026.

Fiebach, C. J., Gruber, T., \& Supp, G. G. (2005). Neuronal mechanisms of repetition priming in occipito-temporal cortex: Spatiotemporal evidence from functional magnetic resonance imaging and electroencephalography. Journal of Neuroscience, 25, 3414-3422.

Gaillard, R., Naccache, L., Pinel, P., Clemenceau, S., Volle, E., Hasboun, D., et al. (2006). Direct intracranial, fMRI, and lesion evidence for the causal role of left inferotemporal cortex in reading. Neuron, 50, 191-204.

Geschwind, N. (1965). Disconnexion syndromes in animals and man. Brain, 88, 237-294.

Givre, S. J., Schroeder, C. E., \& Arezzo, J. C. (1994). Contribution of extrastriate area $\mathrm{v} 4$ to the surface-recorded flash vep in the awake macaque. Vision Research, 34, 415-428.

Glover, G. H. (1999). Deconvolution of impulse response in event-related BOLD fMRI. Neuroimage, 9, 416-429.

Göbel, S., Walsh, V., \& Rushworth, M. F. S. (2001). The mental number line and the human angular gyrus. Neuroimage, 14, 1278-1289.

Gough, P. M., Nobre, A. C., \& Devlin, J. T. (2005). Dissociating linguistic processes in the left inferior frontal cortex with transcranial magnetic stimulation. Journal of Neuroscience, 25, 8010-8016.

Grill-Spector, K., Kushnir, T., Edelman, S., Avidan, G., Itzchak, Y., \& Malach, R. (1999). Differential processing 
of objects under various viewing conditions in the human lateral occipital complex. Neuron, 24, 187-203.

Haxby, J. V., Gobbini, M. I., Furey, M. L., Ishai, A., Schouten, J. L., \& Pietrini, P. (2001). Distributed and overlapping representations of faces and objects in ventral temporal cortex. Science, 293, 2425-2430.

Haxby, J. V., Hoffman, E. A., \& Gobbini, M. I. (2000). The distributed human neural system for face perception. Trends in Cognitive Sciences, 4, 223-233.

Haxby, J. V., Horwitz, B., Ungerleider, L. G., Maisog, J. M., Pietrini, P., \& Grady, C. L. (1994). The functional-organization of human extrastriate cortex-A PET-RCBF study of selective attention to faces and locations. Journal of Neuroscience, 14. 6336-6353.

Hillis, A. E., Newhart, M., Heidler, J., Barker, P., Herskovits, E., \& Degaonkar, M. (2005). The roles of the "visual word form area" in reading. Neuroimage, 24, 548-559.

Ilmoniemi, R. J., Virtanen, J., Ruohonen, J., Karhu, J., Aronen, H. J., Näätänen, R., et al. (1997). Neuronal responses to magnetic stimulation reveal cortical reactivity and connectivity. NeuroReport, 8, 3537-3540.

Jenkinson, M., Bannister, P., Brady, M., \& Smith, S. (2002). Improved optimization for the robust and accurate linear registration and motion correction of brain images. Neuroimage, 17, 825-841.

Jenkinson, M., \& Smith, S. (2001). A global optimisation method for robust affine registration of brain images. Medical Image Analysis, 5, 143-156.

Joseph, J. E., Cerullo, M. A., Farley, A. B., Steinmetz, N. A., \& Mier, C. R. (2006). fMRI correlates of cortical specialization and generalization for letter processing. Neuroimage, 32, 806-820.

Juan, C.-H., \& Walsh, V. (2003). Feedback to V1: A reverse hierarchy in vision. Experimental Brain Research, 150, 259-263.

Kanwisher, N., McDermott, J., \& Chun, M. M. (1997). The fusiform face area: A module in human extrastriate cortex specialized for face perception. Journal of Neuroscience, 17, 4302-4311.

Kronbichler, M., Hutzler, F., Wimmer, H., Mair, A., Staffen, W., \& Ladurner, G. (2004). The visual word form area and the frequency with which words are encountered: Evidence from a parametric fMRI study. Neuroimage, 21, 946-953.

Kuo, W. J., Yeh, T. C., Lee, C. Y., Wu, Y. T., Chou, C. C., Ho, L. T., et al. (2003). Frequency effects of Chinese character processing in the brain: An event-related fMRI study. Neuroimage, 18, 720-730.

Leff, A. P., Crewes, H., Plant, G. T., Scott, S. K., Kennard, C., \& Wise, R. J. S. (2001). The functional anatomy of single-word reading in patients with hemianopic and pure alexia. Brain, 124, 510-521.

Loftus, G. R., \& Masson, M. E. J. (1994). Using confidence intervals in within-subject designs. Psychonomic Bulletin E Review, 1, 476-490.

Malach, R., Reppas, J. B., Benson, R. R., Kwong, K. K., Jiang, H. Kennedy, W. A., et al. (1995). Object-related activity revealed by functional magnetic-resonance-imaging in human occipital cortex. Proceedings of the National Academy of Sciences, U.S.A., 92, 8135-8139.

Manenti, R., Cappa, S. F., Rossini, P. M., \& Miniussi, C. (2008). The role of the prefrontal cortex in sentence comprehension: An rTMS study. Cortex, 44, 337-344.

Maunsell, J. H. R., \& Gibson, J. R. (1992). Visual response latencies in striate cortex of the macaque monkey. Journal of Neurophysiology, 68, 1332-1344.

McCandliss, B. D., Cohen, L., \& Dehaene, S. (2003). The visual word form area: Expertise for reading in the fusiform gyrus. Trends in Cognitive Sciences, 7, 293-299.
McCandliss, B. D., Posner, M. I., \& Givon, T. (1997). Brain plasticity in learning visual words. Cognitive Psychology, 33, 88-110.

Mechelli, A., Gorno-Tempini, M. L., \& Price, C. J. (2003). Neuroimaging studies of word and pseudoword reading: Consistencies, inconsistencies, and limitations. Journal of Cognitive Neuroscience, 15, 260-271.

Moore, C. J., \& Price, C. J. (1999). Three distinct ventral occipitotemporal regions for reading and object naming. Neuroimage, 10, 181-192.

Nobre, A. C., Allison, T., \& McCarthy, G. (1994). Word recognition in the human inferior temporal lobe. Nature, 372, 260-263.

O'Shea, J., Johansen-Berg, H., Trief, D., Gobel, S., \& Rushworth, M. F. S. (2007). Functionally specific in human premotor reorganization cortex. Neuron, 54, 479-490.

Pammer, K., Hansen, P. C., Kringelbach, M. L., Holliday, I., Barnes, G., Hillebrand, A., et al. (2004). Visual word recognition: The first half second. Neuroimage, 22, 1819-1825.

Pascual-Leone, A., Bartres-Faz, D., \& Keenan, J. P. (1999). Transcranial magnetic stimulation: Studying the brain-behaviour relationship by induction of "virtual lesions". Philosophical Transactions of the Royal Society of London, Series B, Biological Sciences, 354, 1229-1238.

Pascual-Leone, A., Vallssole, J., Wassermann, E. M., \& Hallett, M. (1994). Responses to rapid-rate transcranial magnetic stimulation of the human motor cortex. Brain, $117,847-858$

Paus, T., Jech, R., Thompson, C. J., Comeau, R., Peters, T., \& Evans, A. C. (1997). Transcranial magnetic stimulation during positron emission tomography: A new method for studying connectivity of the human cerebral cortex. Journal of Neuroscience, 17, 3178-3184.

Philipose, L. E., Gottesman, R. F., Newhart, M., Kleinman, J. T., Herskovits, E. H., Pawlak, M. A., et al. (2007). Neural regions essential for reading and spelling of words and pseudowords. Annals of Neurology, 62, 481-492.

Pitcher, D., Charles, L., Devlin, J. T., Walsh, V., \& Duchaine, B. (2009). Triple dissociation of faces, bodies, and objects in extrastriate cortex. Current Biology, 19, 319-324.

Pitcher, D., Garrido, L., Walsh, V., \& Duchaine, B. C. (2008) Transcranial magnetic stimulation disrupts the perception and embodiment of facial expressions. Journal of Neuroscience: The Official Journal of the Society for Neuroscience, 28, 8929-8933.

Pitcher, D., Walsh, V., Yovel, G., \& Duchaine, B. (2007). TMS evidence for the involvement of the right occipital face area in early face processing. Current Biology, 17, 1568-1573.

Price, C. J., \& Devlin, J. T. (2003). The myth of the visual word form area. Neuroimage, 19, 473-481.

Price, C. J., \& Devlin, J. T. (2004). The pro and cons of labelling a left occipitotemporal region: "The visual word form area". Neuroimage, 22, 477-479.

Price, C. J., \& Friston, K. J. (2005). Functional ontologies for cognition: The systematic definition of structure and function. Cognitive Neuropsychology, 22, 262-275.

Price, C. J., McCrory, E., Noppeney, U., Mechelli, A., Moore, C. J., Biggio, N., et al. (2006). How reading differs from object naming at the neuronal level. Neuroimage, 29, 643-648.

Price, C. J., Wise, R. J. S., \& Frackowiak, R. S. J. (1996). Demonstrating the implicit processing of visually presented words and pseudowords. Cerebral Cortex, 6, 62-70.

Pulvermüller, F., Huss, M., Kherif, F., Moscoso del Prado Martin, F., Hauk, O., \& Shtyrov, Y. (2006). Motor cortex 
maps articulatory features of speech sounds. Proceedings of the National Academy of Sciences, U.S.A., 103, 7865-7870.

Richmond, B. J., Optican, L. M., Podell, M., \& Spitzer, H. (1987). Temporal encoding of two-dimensional patterns by single units in primate inferior temporal cortex: 1. Response characteristics. Journal of Neurophysiology, 57, $132-146$.

Rothwell, J. C. (1997). Techniques and mechanisms of action of transcranial stimulation of the human motor cortex. Journal of Neuroscience Methods, 74, 113-122.

Rumsey, J. M., Horwitz, B., Donohue, B. C., Nace, K., Maisog, J. M., \& Andreason, P. (1997). Phonological and orthographic components of word recognition-A PET-rCBF study. Brain, 120, 739-759.

Rushworth, M. F. S., Ellison, A., \& Walsh, V. (2001). Complementary localization and lateralization of orienting and motor attention. Nature Neuroscience, 4, 656-661.

Sack, A. T. (2006). Transcranial magnetic stimulation, causal structure-function mapping and networks of functional relevance. Current Opinion in Neurobiology, 16, 593-599.

Sack, A. T., Cohen Kadosh, R., Schuhmann, T., Moerel, M., Walsh, V., \& Goebel, R. (2009). Optimizing functional accuracy of TMS in cognitive studies: A comparison of methods. Journal of Cognitive Neuroscience, 21, 207-221.

Salmelin, R., Service, E., Kiesila, P., Uutela, K., \& Salonen, O. (1996). Impaired visual word processing in dyslexia revealed with magnetoencephalography. Annals of Neurology, 40, 157-162.

Sandrini, M., Rossini, P. M., \& Miniussi, C. (2008). Lateralized contribution of prefrontal cortex in controlling task-irrelevant information during verbal and spatial working memory tasks: rTMS evidence. Neuropsychologia, 46, 2056-2063.

Schluter, N. D., Rushworth, M. F. S., Mills, K. R., \& Passingham, R. E. (1999). Signal-, set-, and movement-related activity in the human premotor cortex. Neuropsychologia, 37, 233-243.

Schroeder, C. E., Mehta, A. D., \& Givre, S. J. (1998). A spatiotemporal profile of visual system activation revealed by current source density analysis in the awake macaque. Cerebral Cortex, 8, 575-592.

Sergent, J., Ohta, S., \& Macdonald, B. (1992). Functional neuroanatomy of face and object processing-A positron emission tomography study. Brain, 115, 15-36.

Skarratt, P. A., \& Lavidor, M. (2006). Magnetic stimulation of the left visual cortex impairs expert word recognition. Journal of Cognitive Neuroscience, 18, 1749-1758.

Sparing, R., Buelte, D., Meister, I. G., Paus, T., \& Fink, G. R. (2008). Transcranial magnetic stimulation and the challenge of coil placement: A comparison of conventional and stereotaxic neuronavigational strategies. Human Brain Mapping, 29, 82-96.
Starrfelt, R., \& Gerlach, C. (2007). The visual what for area: Words and pictures in the left fusiform gyrus. Neuroimage, 35, 334-342.

Tarkiainen, A., Helenius, P., Hansen, P. C., Cornelissen, P. L., \& Salmelin, R. (1999). Dynamics of letter string perception in the human occipitotemporal cortex. Brain, 122, 2119-2131.

Taylor, P. C. J., Nobre, A. C., \& Rushworth, M. F. S. (2007). FEF TMS affects visual cortical activity. Cerebral Cortex, 17, 391-399.

Terao, Y., Ugawa, Y., Suzuki, M., Sakai, K., Hanajima, R., GembaShimizu, K., et al. (1997). Shortening of simple reaction time by peripheral electrical and submotor-threshold magnetic cortical stimulation. Experimental Brain Research, 115, 541-545.

Ulrich, R., \& Miller, J. (1994). Effects of truncation on reaction time analysis. Journal of Experimental Psychology: General, 123, 34-80.

Walsh, V., \& Cowey, A. (2000). Transcranial magnetic stimulation and cognitive neuroscience. Nature Reviews Neuroscience, 1, 73-79.

Walsh, V., Ellison, A., Battelli, L., \& Cowey, A. (1998). Task-specific impairments and enhancements induced by magnetic stimulation of human visual area V5. Proceedings of the Royal Society of London, Series B, Biological Sciences, 265, 537-543.

Warrington, E. K., \& Shallice, T. (1980). Word-form dyslexia. Brain, 103, 99-112.

Wassermann, E. M. (1998). Risk and safety of repetitive transcranial magnetic stimulation: Report and suggested guidelines from the International Workshop on the Safety of Repetitive Transcranial Magnetic Stimulation, June 5-7, 1996. Electroencephalography and Clinical Neurophysiology —Evoked Potentials, 108, 1-16.

Wassermann, E. M., Grafman, J., Berry, C., Hollnagel, C., Wild, K., Clark, K., et al. (1996). Use and safety of a new repetitive transcranial magnetic stimulator. Electroencephalography and Clinical NeurophysiologyElectromyography and Motor Control, 101, 412-417.

Woolrich, M., Ripley, B., Brady, J., \& Smith, S. M. (2001). Temporal autocorrelation in univariate linear modelling of fMRI data. Neuroimage, 14, 1370-1386.

Wright, N., Mechelli, A., Noppeney, U., Veltman, D., Rombouts, S., Glensman, J., et al. (2008). Selective activation around the left occipito-temporal sulcus for words relative to pictures: Individual variability or false positives? Human Brain Mapping, 29, 986-1000.

Yousry, T. A., Schmid, U. D., Alkadhi, H., Schmidt, D., Peraud, A., Buettner, A., et al. (1997). Localization of the motor hand area to a knob on the precentral gyrus. A new landmark. Brain, 120, 141-157. 